\title{
SYNTHESIS AND ANTIMICROBIAL ACTIVITIES OF SOME 4-(6-METHYL-2- NAPHTHYL)-5,6-DIHYDRO-6-(SUBSTITUTED PHENYL)-4H-1,3-OXAZINE-2-AMINES
}

\author{
Ganesamoorthy Thirunarayanan ${ }^{1}$, Krishnamoorthy Guna Sekar ${ }^{2}$ \\ ${ }^{1}$ Department of Chemistry, Annamalai University, Annamalainagar-608 002, India. \\ ${ }^{2}$ Department of Chemistry, National College, Tiruchirappalli-620 001, India \\ E-mail:drkgsekar@yahoo.co.in
}

\begin{abstract}
Some 4-(6-methyl-2-naphthyl)-5,6-dihydro-6-(substituted phenyl)-4H-1,3-oxazine-2amines have been synthesised by hydroxyapatite catalyzed solvent-free cyclization of 6methyl-2-naphthyl chalcones and urea under microwave irradiation. The yields of the oxazine were more than $85 \%$. The synthesised oxazine amines have been characterized by their physical constants, analytical and spectroscopic data. The antimicrobial activities of all prepared 4-(6-methyl-2-naphthyl)-5, 6-dihydro-6-(substituted phenyl)-4H-1,3oxazine-2-amines have been evaluated using Bauer-Kirby disc diffusion method with a variety of bacterial and fungal strains.
\end{abstract}

Keywords: Oxazine-2-amines, Enones, Hydroxyapatite, Green synthesis, Antimicrobial activities.

\section{INTRODUCTION}

The unsaturated 1,3-oxazine and their derivatives are important bio-active six membered heterocyclic compounds which possess one oxygen and one nitrogen atom $[1,2]$. Oxazines exists many isomeric structures such as 1,2 or 1,3 or 1, 4 oxazines [3] depend upon the relative position of these tow atoms and the double bond. These oxazines were medicinally important due to the presence of oxygen, nitrogen heteroatoms along with double bonds in their structural moieties [4]. The important medicinal activities of these oxazines are anti-bacterial, anti-fungal [4-6], anti-plasmodial [7], anticancer [8], anti-depressants [9], cytotoxicity [10], anti-osteoplastic [11], anti- tumour [12], anti-oxidant [13], anti-tuberculosis [14], antineoplastic [15], antagonists [16], anti- inflammatory [17], anti-infectants [18], IKB kinase beta [19] and PTP-1B inhibition [20]. These oxazine derivatives were applied for improving the super resolution microscope [21], synthesis of eosinophils [22], identification and separation of neutrophils [23]. Many oxazine derivatives were used as a dyes [24]. Nowadays scientists, organic chemists are interested for solvent-free synthesis [4, 25-31] of organic substrates. Numerous solvent assisted and solvent-free synthetic methods were available for synthesis of oxazine derivatives [4, 25-27, 32,]. Hetero Diels-Alder reaction [4], ring closure [33], Betti base induced condensation [27], Mannich type condensation-cyclization [5] and cyclization of chalcones [6] were used for synthesis of oxazine derivatives [25] have synthesised some benzoxazine/ oxazine fused isoquinolines and naphthyridines by solvent- 
free method [25]. They have synthesised some 1,3-oxazine derivatives by solventassisted method from chalcones and urea [6]. More than $75 \%$ yield of dihydro-2H-benzoand naphtho-1, 3-oxazine derivatives were prepared by Mathew et al.[5] using ecofriendly method. Efficient synthesis of some 1,3-oxazine-4-thiones were synthesised by $\mathrm{N}$ methyl imidazole promoted solvent-free conditions. Sapkal et al., have studied the role of ammonium acetate for solvent-free synthesis of 1, 3-disubstituted-2,3-dihydro$1 \mathrm{H}$-naphyl oxazines [27]. Within the above view, there is no information available in the literature for the solvent-free synthesis of oxazine amine-2-derivatives including 4-(6methyl-2-naphthyl)-6-(substituted phenyl) $4 \mathrm{H}-1,3$-oxazine-2-amines by cyclization of enones. Therefore the authors have taken effort to synthesized some 4-(6-methyl-2naphthyl)-6-(substituted phenyl) 4H-1,3oxazine-2-amines by solvent-free cyclization of enones and urea under microwave irradiation and studied the antimicrobial activities.

\section{EXPERIMENTAL}

\subsection{GENERAL}

All chemicals were used in this study were purchased from Sigma-Aldrich and Merck Chemical companies. Mettler FP51 melting point apparatus was used for determining the melting point of all synthesized oxazine-2amines in open glass capillaries and are uncorrected. The AVATAR-300 Fourier transform spectrophoto meter was used for recording infrared spectra $(\mathrm{KBr}, 4000-400$ $\mathrm{cm}-1$ ) of all oxazines in $\mathrm{KBr}$ disc. The Bruker AV400 series type NMR spectrometer was utilized for recording NMR spectra of all oxazines, operating at $400 \mathrm{MHz}$ for $1 \mathrm{H}$ and $100 \mathrm{MHz}$ for $13 \mathrm{C}$ spectra in $\mathrm{CDCl}_{3}$ solvent using TMS as internal standard. Mass spectra of all synthesised oxazines were recorded on SHIMADZU mass spectrometer using chemical ionization technique.

\subsection{PREPARATION OF HYDROXYAPATITE AND 6-METHYL-2-NAPHTHYL CHALCONES}

Hydroxyapatite catalyst and 6-methyl-2naphthyl chalcones were prepared according to literature procedure [34].

\subsection{SYNTHESIS OF 4-(6-METHYL-2- NAPHTYL)-5,6-DIHYDRO-6- (SUBSTITUTED PHENYL)-4H-1,3- OXAZINE-2-AMINES}

An appropriate equi-molar quantities of 6methyl-2-naphtyl chalcones $(2 \mathrm{mmol})$, urea (2 $\mathrm{mmol}$ ) and $0.4 \mathrm{~g}$ of hydroxyapatite were taken in a $50 \mathrm{~mL}$ beaker, closed with the lid. This mixture was subjected to microwave irradiation for 2-4 minutes at $650 \mathrm{~W}$ (Samsung Grill, GW73BD Microwave oven, $230 \mathrm{~V} \mathrm{~A} / \mathrm{c}, 50 \mathrm{~Hz}, 2450 \mathrm{~Hz}, 100-750 \mathrm{~W}$ (IEC705), (Scheme 1). After completion of the reaction, dichloromethane $(20 \mathrm{~mL})$ was added, followed by simple filtration. The solution was concentrated and purified by recrystallization. The synthesized oxazines were characterized by their physical constants, IR, $1 \mathrm{H}$ and $13 \mathrm{C}$ NMR and Mass spectral data.

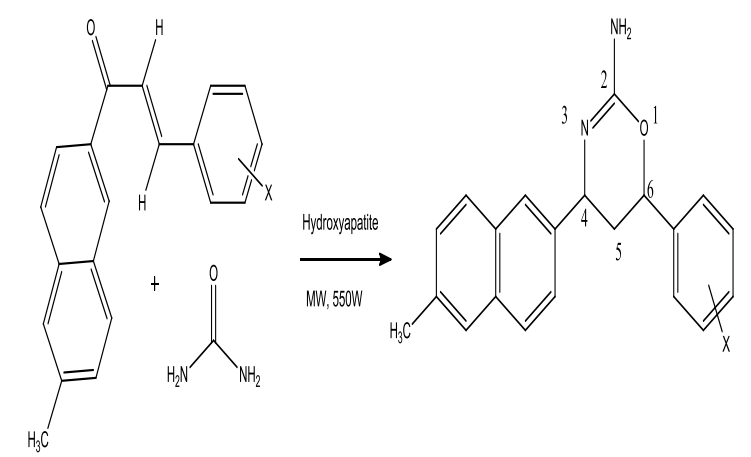

$\mathrm{X}=\mathrm{H}, 3-\mathrm{NH}_{2}, 4-\mathrm{NH}_{2}, 3 \cdot-\mathrm{Br}, 3 \cdot \mathrm{Cl}, 4-\mathrm{Cl}, 4 \cdot \mathrm{N}\left(\mathrm{CH}_{3,2}, 4 \cdot \mathrm{OH}, 4 \cdot-\mathrm{OCH}_{3}, 4-\mathrm{CH}_{3}, 2 \cdot \mathrm{NO}_{2}, 3 \cdot \mathrm{NO}_{2}, 4 \cdot-\mathrm{NO}_{2}\right.$

Scheme. 1. Synthesis of 4-(6-methyl-2-naphtyl)-5,6-dihydro-6(substituted phenyl)-4H-1,3-oxazine-2-amines. 


\section{RESULTS AND DISCUSSION}

The authors attempts made to synthesize oxazine-2-amine derivatives by cyclization of chalcones possess electron with-drawing as well as electron donating group as substituents, urea and in the presence of a catalyst hydroxyapatite using microwave irradiation. Hence the authors have synthesized some substituted 1,3-oxazine derivatives by the cyclization of $2 \mathrm{mmol}$ of 6-methyl-2naphthyl chalcones, $2 \mathrm{mmol}$ of urea under microwave irradiation with $0.4 \mathrm{~g}$ of hydroxyapatite catalyst at 550W for 4-6 minutes (Scheme 1). During the course of this reaction hydroxyapatite catalyses cyclization between 6-methyl-2-naphthyl chalcones and urea followed by rearrangement gave the 1 , 3 -oxazine amines. The yield of the oxazine in this reaction is more than $80 \%$. The 6methyl-2-naphthyl chalcones containing electron donating substituent $\left(\mathrm{OCH}_{3}\right)$ gave higher yields than electron-withdrawing (halogens, $\mathrm{NO}_{2}$ ) substituents. Further we have investigated this cyclization reaction with equimolar quantities of the styryl 6-methyl-2naphthyl ketone (entry 1) and urea under the same condition as above. In this reaction the obtained yield was $90 \%$. The effect of catalyst on this reaction was studied by varying the catalyst quantity from $0.1 \mathrm{~g}$ to $1 \mathrm{~g}$. As the catalyst quantity is increased from 0.1 $\mathrm{g}$ to $0.4 \mathrm{~g}$, the percentage of yield of product is increased from 84 to $90 \%$. Further increase in the catalyst amount beyond $0.4 \mathrm{~g}$, there is no significant increase in the percentage of the product. The effect of catalyst loading is shown in Figure 1. The optimum quantity of catalyst loading was found to be $0.4 \mathrm{~g}$. The physical constants, analytical and mass spectral data are summarized in Table 1. The effect of solvents on the yield was also studied with methanol, ethanol, dichloromethane and tetrahydrofuran from each component of the catalyst (entry 1). Similarly the effect of microwave irradiation was studied on each component of the catalyst. The effect of solvents on the yield of oxazine derivatives was presented in Table 2. From the table highest yield of oxazine obtained from the cyclization of 6-methyl-2-naphthyl chalcones and urea with the catalyst hydroxyapatite in microwave irradiation.

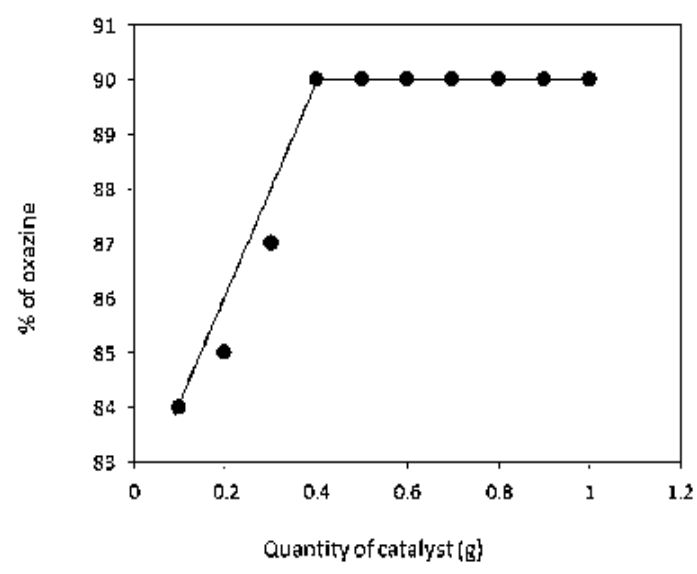

Fig.1. Effect of catalyst loading.

The infrared and nmr spectroscopic data of selective oxazine-2-amine derivatives are summarized as follows:

4-(6-Methyl-2-naphthyl)-5,6-dihydro-6phenyl-4H-1,3-oxazine-2-amine (1): FTIR $(\mathrm{KBr}): 3538(\mathrm{NH}), 1587(\mathrm{C}=\mathrm{N}), 1212$ (C-OC) $\mathrm{cm}-1.1 \mathrm{H} \mathrm{NMR}\left(\mathrm{CDCl}_{3}-\mathrm{d} 6, \mathrm{TMS}\right) \delta$ : $2.321\left(\mathrm{~s}, 1 \mathrm{H}, \mathrm{NH}_{2}\right), 2.655(\mathrm{dd}, 1 \mathrm{H}, \mathrm{H} 4)$, 2.345 (dd, 1H, H5), 2.217 (dd, 1H, H5'), 4.256 (dd, $1 \mathrm{H}, \mathrm{H} 6), 3.575$ (s, 3H, $\mathrm{CH}_{3}$ ), 6.521-7.378 (m, 11H, Ar-H) ppm.; 13C NMR ( $\left.\mathrm{CDCl}_{3}-\mathrm{d} 6, \mathrm{TMS}\right) \delta$ : 165.34 (C2), 52.76 (C4), 47.34 (C5), 65.68 (C6), 25.98 $\left(\mathrm{CH}_{3}\right), 125.34-142.87$ (Ar-C) ppm

4-(6-Methyl-2-naphthyl)-5,6-dihydro-6-(3aminoyphenyl)-4H-1,3-oxazine-2-amine (2): FTIR (KBr): $3560 \quad(\mathrm{NH}), \quad 1615(\mathrm{C}=\mathrm{N})$, 1224(C-O-C) cm-1. 1H NMR ( $\mathrm{CDCl}_{3}$-d6, 
TMS) $\delta: 2.229\left(\mathrm{~s}, 1 \mathrm{H}, \mathrm{NH}_{2}\right), 2.532(\mathrm{dd}, 1 \mathrm{H}$, H4), 2.443(dd, 1H, H5), 2.211(dd, 1H, H5'), $3.353(\mathrm{dd}, 1 \mathrm{H}, \mathrm{H} 6), 3.243\left(\mathrm{~s}, 3 \mathrm{H}, \mathrm{CH}_{3}\right)$, 6.234-7.265(m, 10H, Ar-H) ppm. 13C NMR ( $\left.\mathrm{CDCl}_{3}-\mathrm{d} 6, \mathrm{TMS}\right) \delta: \quad 164.32(\mathrm{C} 2)$, 51.36 (C4), 47.26(C5), $66.54 \quad$ (C6), 24.65 $\left(\mathrm{CH}_{3}\right), 126.54-139.67$ (Ar-C)ppm.

4-(6-Methyl-2-naphthyl)-5,6-dihydro-6-(4aminophenyl)-4H-1,3-oxazine-2-amine (3): FTIR $(\mathrm{KBr}): \quad 3555(\mathrm{NH}), \quad$ 1620 $(\mathrm{C}=\mathrm{N})$, 1212(C-O-C) cm-1. 1H NMR $\left(\mathrm{CDCl}_{3}-\mathrm{d} 6\right.$, TMS) $\delta: 2.223\left(\mathrm{~s}, 1 \mathrm{H}, \mathrm{NH}_{2}\right), 2.556(\mathrm{dd}, 1 \mathrm{H}$, H4), $2.454(\mathrm{dd}, 1 \mathrm{H}, \mathrm{H} 5), 2.265(\mathrm{dd}, 1 \mathrm{H}$, $\mathrm{H}^{\prime}$ ), 4.371(dd, 1H, H6), 3.234(s, 3H, $\left.\mathrm{CH}_{3}\right)$, 6.234-7.276 (m, 10H, Ar-H) ppm. 13C NMR ( $\left.\mathrm{CDCl}_{3}-\mathrm{d} 6, \mathrm{TMS}\right) \delta$ : $164.43(\mathrm{C} 2)$, 51.76 (C4), 47.35(C5), 66.76 (C6), 24.65( $\left(\mathrm{CH}_{3}\right), 126.34-139.89$ (Ar-C)ppm.

4-(6-Methyl-2-naphthyl)-5,6-dihydro-6-(3bromophenyl)-4H-1,3-oxazine-2-amine (4): FTIR(KBr): $\quad 3540(\mathrm{NH}), \quad 1612(\mathrm{C}=\mathrm{N})$, 1224(C-O-C) cm-1. 1H NMR ( $\mathrm{CDCl}_{3}-\mathrm{d} 6$, TMS $) \delta: 2.218\left(\mathrm{~s}, 1 \mathrm{H}, \mathrm{NH}_{2}\right), 2.324(\mathrm{dd}, 1 \mathrm{H}$, $\mathrm{H} 4), 2.265 \mathrm{dd}, 1 \mathrm{H}, \mathrm{H} 5), 2.267$ (dd, 1H, H5'), 4.360(dd, 1H, H6), $3.334\left(\mathrm{~s}, 3 \mathrm{H}, \mathrm{CH}_{3}\right)$, 6.234-7.267(m, 10H, Ar-H) ppm. 13C NMR ( $\left.\mathrm{CDCl}_{3}-\mathrm{d} 6, \mathrm{TMS}\right) \delta$ : 164.46 (C2), 51.56 (C4), 47.65(C5), 66.76 (C6), 25.33( $\left(\mathrm{CH}_{3}\right), 124.87-139.90$ (Ar-C)ppm.

4-(6-Methyl-2-naphthyl)-5,6-dihydro-6-(3chlorophenyl)-4H-1,3-oxazine-2-amine (5): FTIR $(\mathrm{KBr}): \quad 3550(\mathrm{NH}), \quad 1615(\mathrm{C}=\mathrm{N})$, 1230(C-O-C) cm-1. 1H NMR $\left(\mathrm{CDCl}_{3}\right.$-d6, TMS) $\delta: 2.256\left(\mathrm{~s}, 1 \mathrm{H}, \mathrm{NH}_{2}\right), 2.332(\mathrm{dd}, 1 \mathrm{H}$, H4), 2.416(dd, 1H, H5), 2.265 (dd, 1H, $\mathrm{H}^{\prime}$ ) $, 4.376(\mathrm{dd}, 1 \mathrm{H}, \mathrm{H} 6), 3.142\left(\mathrm{~s}, 3 \mathrm{H}, \mathrm{CH}_{3}\right)$, 6.256-7.276 (m, 10H, Ar-H) ppm. 13C NMR ( $\left.\mathrm{CDCl}_{3}-\mathrm{d} 6, \mathrm{TMS}\right) \delta: \quad 164.67(\mathrm{C} 2)$, 51.98 (C4), 47.02(C5), 66.47 (C6), 24.12( $\left(\mathrm{CH}_{3}\right), 126.65-139.87$ (Ar-C)ppm.
4-(6-Methyl-2-naphthyl)-5,6-dihydro-6-(4chlorophenyl)-4H-1,3-oxazine-2-amine (6): FTIR(KBr): $\quad 3550(\mathrm{NH}), \quad 1605(\mathrm{C}=\mathrm{N})$, 1223(C-O-C) cm-1. $1 \mathrm{H}$ NMR $\left(\mathrm{CDCl}_{3}-\mathrm{d} 6\right.$,

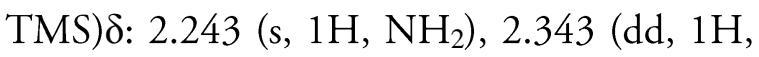
H4), 2.422(dd, $1 \mathrm{H}, \mathrm{H} 5), 2.232\left(\mathrm{dd}, 1 \mathrm{H}, \mathrm{H}^{\prime}\right.$ ), 4.368(dd, 1H, H6), 2.932(s, 3H, $\left.\mathrm{CH}_{3}\right)$, 6.243-7.276 (m, 10H, Ar-H) ppm. 13C NMR $\left(\mathrm{CDCl}_{3}-\mathrm{d} 6, \mathrm{TMS}\right) \delta: \quad 164.56(\mathrm{C} 2)$, 51.87 (C4), 47.87(C5), 66.72 (C6), 24.11( $\left(\mathrm{CH}_{3}\right), 126.65-139.87(\mathrm{Ar}-\mathrm{C}) \mathrm{ppm}$.

4-(6-Methyl-2-naphthyl)-5,6-dihydro-6-(4dimethylaminophenyl)-4H-1,3-oxazine-2amine (7): FTIR $(\mathrm{KBr}): \quad 3530(\mathrm{NH})$, 1592(C=N), 1206(C-O-C) cm-1. 1H NMR $\left(\mathrm{CDCl}_{3}-\mathrm{d} 6, \mathrm{TMS}\right) \delta: \quad 2.211\left(\mathrm{~s}, 1 \mathrm{H}, \mathrm{NH}_{2}\right)$, $2.330(\mathrm{dd}, 1 \mathrm{H}, \mathrm{H} 4), 2.420(\mathrm{dd}, 1 \mathrm{H}, \mathrm{H} 5)$, $2.232\left(\mathrm{dd}, 1 \mathrm{H}, \mathrm{H} 5^{\prime}\right), 4.328(\mathrm{dd}, 1 \mathrm{H}, \mathrm{H} 6)$, 3.203(s, 6H, $\left.\mathrm{N}\left(\mathrm{CH}_{3}\right) 2\right), 3.154\left(\mathrm{~s}, 3 \mathrm{H}, \mathrm{CH}_{3}\right)$, 26.265-7.218 (m, 10H, Ar-H) ppm. 13C NMR ( $\left.\mathrm{CDCl}_{3}-\mathrm{d} 6, \mathrm{TMS}\right) \delta: 164.80$ (C2), 51.45 (C4), 47.78(C5), 66.98 (C6), $44.25\left(\mathrm{~N}\left(\mathrm{CH}_{3}\right) 2\right), \quad 24.55\left(\mathrm{CH}_{3}\right), \quad 126.19-$ 139.76(Ar-C)ppm.

4-(6-Methyl-2-naphthyl)-5,6-dihydro-6-(4hydroxyphenyl)-4H-1,3-oxazine-2-amine (8): FTIR $(\mathrm{KBr}): \quad 3540(\mathrm{NH}), \quad 1615(\mathrm{C}=\mathrm{N})$, 1211(C-O-C) cm-1. 1H NMR ( $\mathrm{CDCl}_{3}-\mathrm{d} 6$,

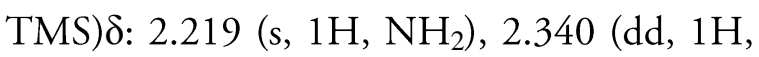
H4), 2.454(dd, 1H, H5), 2.287(dd, 1H, H5'), $4.376(\mathrm{dd}, 1 \mathrm{H}, \mathrm{H} 6), 3.198\left(\mathrm{~s}, 3 \mathrm{H}, \mathrm{CH}_{3}\right)$, 6.234-7.254(m, 10H, Ar-H) ppm. 13C NMR ( $\left.\mathrm{CDCl}_{3}-\mathrm{d} 6, \mathrm{TMS}\right) \delta$ : 164.64 (C2), 51.54 (C4), 47.65(C5), 63.76 (C6), 44.76 $\left(\mathrm{N}\left(\mathrm{CH}_{3}\right) 2\right), \quad 24.67\left(\mathrm{~s}, \quad 3 \mathrm{H}, \quad \mathrm{CH}_{3}\right), \quad 126.64-$ 139.76(Ar-C)ppm.

4-(6-Methyl-2-naphthyl)-5,6-dihydro-6-(4methoxyphenyl)-4H-1,3-oxazine-2-amine (9): FTIR $(\mathrm{KBr}): \quad 3540(\mathrm{NH}), \quad 1624(\mathrm{C}=\mathrm{N})$, 1218(C-O-C) cm-1. 1H NMR ( $\mathrm{CDCl}_{3}-\mathrm{d} 6$, 
TMS)反: $2.217\left(\mathrm{~s}, 1 \mathrm{H}, \mathrm{NH}_{2}\right), 2.334(\mathrm{dd}, 1 \mathrm{H}$, H4), $2.454(\mathrm{dd}, 1 \mathrm{H}, \mathrm{H} 5), 2.265\left(\mathrm{dd}, 1 \mathrm{H}, \mathrm{H} 5^{\prime}\right.$ ), $4.327(\mathrm{dd}, 1 \mathrm{H}, \mathrm{H} 6), 4.233\left(\mathrm{~s}, 3 \mathrm{H}, \mathrm{OCH}_{3}\right)$, $3.326\left(\mathrm{~s}, 3 \mathrm{H}, \mathrm{CH}_{3}\right), 6.233-7.253(\mathrm{~m}, 10 \mathrm{H}, \mathrm{Ar}-$ H) ppm. 13C NMR ( $\mathrm{CDCl}_{3}-\mathrm{d} 6$, TMS $) \delta$ : 164.54 (C2), 51.26 (C4), 47.37(C5), 66.76 (C6), 64.76(s, 3H, $\left.\mathrm{OCH}_{3}\right), 24.98(\mathrm{~s}, 3 \mathrm{H}$, $\left.\mathrm{CH}_{3}\right)$, 126.32-139.66(Ar-C)ppm.

4-(6-Methyl-2-naphthyl)-5,6-dihydro-6-(4methylphenyl)-4H-1,3-oxazine-2-amine (10): FTIR(KBr): $\quad 3538(\mathrm{NH}), \quad 1616(\mathrm{C}=\mathrm{N})$, 1217(C-O-C) cm-1. 1H NMR ( $\mathrm{CDCl}_{3}-\mathrm{d} 6$,

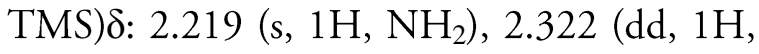
H4), 2.436(dd, $1 \mathrm{H}, \mathrm{H} 5), 2.226\left(\mathrm{dd}, 1 \mathrm{H}, \mathrm{H} 5^{\prime}\right)$, 4.347(dd, 1H, H6), 2.833 (s, 3H, $\left.\mathrm{CH}_{3}\right)$, $2.513\left(\mathrm{~s}, 3 \mathrm{H}, \mathrm{CH}_{3}\right), 6.288-7.272(\mathrm{~m}, 10 \mathrm{H}$, Ar-H) ppm. 13C NMR ( $\left.\mathrm{CDCl}_{3}-\mathrm{d} 6, \mathrm{TMS}\right) \delta$ : 164.78 (C2), 51.89 (C4), 47.45(C5), 66.76 $(\mathrm{C} 6), \quad 25.87\left(\mathrm{CH}_{3}\right), \quad 25.43\left(\mathrm{CH}_{3}\right), \quad 114.65-$ 145.87(Ar-C)ppm.

4-(6-Methyl-2-naphthyl)-5,6-dihydro-6-(2nitrophenyl)-4H-1,3-oxazine-2-amine (11): FTIR(KBr): $\quad 3555(\mathrm{NH}), \quad 1622(\mathrm{C}=\mathrm{N})$, 1214(C-O-C) cm-1. 1H NMR $\left(\mathrm{CDCl}_{3}-\mathrm{d} 6\right.$,

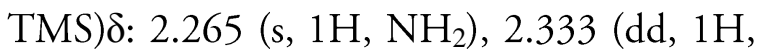
H4), 2.465(dd, $1 \mathrm{H}, \mathrm{H} 5), 2.265(\mathrm{dd}, 1 \mathrm{H}, \mathrm{H} 5$ '), $4.365(\mathrm{dd}, 1 \mathrm{H}, \mathrm{H} 6), 3.164$ (s, $\left.3 \mathrm{H}, \mathrm{CH}_{3}\right)$, 6.243-7.276(m, 10H, Ar-H) ppm. 13C NMR (CDCl $-\mathrm{d} 6, \mathrm{TMS}) \delta: 64.54(\mathrm{C} 2), 51.65$ (C4), 47.87(C5), 66.34 (C6), 26.87(s, 3H, $\left.\mathrm{CH}_{3}\right), 114.65-145.76(\mathrm{Ar}-\mathrm{C}) \mathrm{ppm}$.

4-(6-Methyl-2-naphthyl)-5,6-dihydro-6-(3nitrophenyl)-4H-1,3-oxazine-2-amine (12):

FTIR(KBr): $\quad 3558(\mathrm{NH}), \quad 1622(\mathrm{C}=\mathrm{N})$, 1218(C-O-C) cm-1. 1H NMR ( $\mathrm{CDCl}_{3}-\mathrm{d} 6$, TMS)反: $2.218\left(\mathrm{~s}, 1 \mathrm{H}, \mathrm{NH}_{2}\right), 2.339(\mathrm{dd}, 1 \mathrm{H}$, H4), 2.465(dd, $1 \mathrm{H}, \mathrm{H} 5), 2.242\left(\mathrm{dd}, 1 \mathrm{H}, \mathrm{H} 5^{\prime}\right.$ ), $4.354(\mathrm{dd}, 1 \mathrm{H}, \mathrm{H} 6), 3.056\left(\mathrm{~s}, 3 \mathrm{H}, \mathrm{CH}_{3}\right)$, 6.544-7.265(m, 10H, Ar-H) ppm. 13C NMR $\left(\mathrm{CDCl}_{3}-\mathrm{d} 6, \mathrm{TMS}\right) \delta: \quad$ 164.68(C2),
51.42 (C4), 47.56(C5), 66.87 (C6), 24.78(s, $\left.3 \mathrm{H}, \mathrm{CH}_{3}\right), 124.88-145.98(\mathrm{Ar}-\mathrm{C}) \mathrm{ppm}$.

4-(6-Methyl-2-naphthyl)-5,6-dihydro-6-(4nitrophenyl)-4H-1,3-oxazine-2-amine (13): FTIR $(\mathrm{KBr}): \quad 3555(\mathrm{NH}), \quad 1633(\mathrm{C}=\mathrm{N})$, 1213(C-O-C) cm-1. 1H NMR ( $\mathrm{CDCl}_{3}-\mathrm{d} 6$, TMS)d: $2.220\left(\mathrm{~s}, 1 \mathrm{H}, \mathrm{NH}_{2}\right), 2.336(\mathrm{dd}, 1 \mathrm{H}$, H4), 2.464(dd, 1H, H5), 2.265(dd, 1H, H5'), $4.376(\mathrm{dd}, 1 \mathrm{H}, \mathrm{H} 6), 3.123\left(\mathrm{~s}, 3 \mathrm{H}, \mathrm{CH}_{3}\right)$, 6.536-7.276(m, 10H, Ar-H) ppm. 13C NMR $\left(\mathrm{CDCl}_{3}-\mathrm{d} 6, \mathrm{TMS}\right) \delta: \quad 164.76(\mathrm{C} 2)$, 51.78 (C4), 47.87(C5), 66.37 (C6), 4.78(s, $\left.3 \mathrm{H}, \mathrm{CH}_{3}\right), 124.25-145.998(\mathrm{Ar}-\mathrm{C}) \mathrm{ppm}$.

\subsection{ANTIMICROBIAL ACTIVITIES \\ 3.1.1 ANTIBACTERIAL ACTIVITIES}

Antibacterial activities of all 4-(6-methyl-2naphthyl)-5,6-dihydro-6-(substitutedphenyl) -oxazine-2-amines were evaluated using Kirby-Bauer [35] disc diffusion technique. In each Petri plate about $0.5 \mathrm{~mL}$ of the test bacterial sample was spread uniformly over the solidified Mueller Hinton agar using sterile glass spreader. Then the discs with 5 $\mathrm{mm}$ diameter made up of Whatmann No.1 filter paper, impregnated with the solution of the compound were placed on the medium using sterile forceps. The plates were incubated for $24 \mathrm{~h}$ at $37^{\circ} \mathrm{C}$ by keeping the plates upside down to prevent the collection of water droplets over the medium [36]. After $24 \mathrm{~h}$, the plates were visually examined and the diameter values of the zone of inhibition were measured. Triplicate results were recorded by repeating the same procedure. The anti-bacterial activities of all prepared 4(6-methyl-2-naphthyl)-5,6-dihydro-6-

(substitutedphenyl)-oxazine-2-amines have been evaluated against two gram positive pathogenic strains Staphylococcus aureus, Enterococcus faecalis and four gram negative 
strains Escherichia coli, Klebsiella species, Psuedomonas and Proteus vulgaris. The disc diffusion technique was followed using the Kirby-Bauer [35] method, at a concentration of $250 \mu \mathrm{g} / \mathrm{mL}$. Ampicillin and Streptomycin were used as the standard drugs. The measured anti-bacterial activities of all 4-(6methyl-2-naphthyl)-5,6-dihydro-6-substituted phenyl)-oxazine-2-amines were presented in Table 3. The oxazine-2-amines 4-6 showed maximum zone of inhibition against Escherichia coli, with greater than $20 \mathrm{~mm}$ compared to the oxazines $3,8-10$ are active in $8-12 \mathrm{~mm}$ of zone of inhibition. The oxazines $1,2,7,11-13$ are inactive. The 1,3-oxazine-2amine 6 only effective against $S$. aureus within 20-24 $\mathrm{mm}$ of zone of inhibition. Compounds 7, 11-13 were moderately active within 13-19 $\mathrm{mm}$ of zone of inhibition. The oxazines $1,5,8-10$ were active within $8-12$ $\mathrm{mm}$ of zone of inhibition. The oxazine derivatives 4-6 were more active against Pseudomonas a showing greater than $20 \mathrm{~mm}$ zone of inhibition and the other derivatives 7 , 8 , and 13 were showed the zone of inhibitions between 12-19 $\mathrm{mm}$. The oxazines 2-3, and 11 were showed active with in $8-12 \mathrm{~mm}$ of zone of inhibition. The oxazine-2-amines 6 was effective within $20-24 \mathrm{~mm}$ of zone of inhibition against Klebsiella species. The oxazine derivative 6 was active against $P$. vulgaris greater than $20 \mathrm{~mm}$ of zone of inhibition. The other compounds are less effective. The oxazines 14 and 16 showed activities against E-faecalis when they are screened with 20-24 mm zone of inhibition.

\subsubsection{ANTI-FUNGAL SENSITIVITY ASSAY}

Antifungal sensitivity assay all 4-(6-methyl-2naphthyl)-5,6-dihydro-6-(substituted phenyl)-oxazine-2-amines (entries 1-13) were performed using Kirby-Bauer disc diffusion technique. PDA medium was prepared and sterilized as above. It was poured (ear bearing heating condition) in the Petri-plate which was already filled with $1 \mathrm{~mL}$ of the fungal species. The plate was rotated clockwise and counter clock-wise for uniform spreading of the species. The discs were impregnated with the test solution. The test solution was prepared by dissolving $15 \mathrm{mg}$ of the oxazinez in $1 \mathrm{~mL}$ of DMSO solvent. The medium was allowed to solidify and kept for $24 \mathrm{~h}$. Then the plates were visually examined and the diameter values of zone of inhibition were measured. Triplicate results were recorded by repeating the same procedure. The study of antifungal activities of all 4-(6-methyl-2naphthyl)-5,6-dihydro-6-(substituted phenyl)-oxazine-2-amines has been done with Candida albicans as the fungal strain using the disc diffusion technique and the other two strains Penicillium species and Aspergillus niger, the dilution method was adopted. The drug dilution was kept as $50 \mathrm{~g} / \mathrm{mL}$. Griseofulvin has been taken as the standard drug. The observed antifungal activities of all all 4-(6-methyl-2-naphthyl)-5,6-dihydro-6(substituted phenyl)-oxazine-2-amines were presented in Table 4. The study of antifungal activities of all 4-(6-methyl-2-naphthyl)-5,6dihydro-6-(substitutedphenyl)-oxazine-2amines against $\mathrm{C}$. albicans, showed that the three compounds 11-13 were effective with $20 \mathrm{~mm}$ as the zone of inhibition in $250 \mu \mathrm{g} /$ disc while oxazine-2-amines 5, 6 and 10 were active with 13-19 $\mathrm{mm}$ zone of inhibition and the compound 1,4,8 and 9 were shows least active with $8-12 \mathrm{~mm}$ zone of inhibitions. The oxazine derivatives $5,6,11-13$ were more visible against Penicillum species, in the development of the fungal colony and 2-3 colonies are recorded for the compound 10 . The inhibition of oxazine-2-amines against $A$. niger was more active in five compounds 5, 6, 
11-13. The compounds $2-4$ and 7 were active with 13-19 mm zone of inhibitions. Presence of amino, dimethylamino, methoxy, methyl, dimethyl, chloro, bromo and nitro substituents are responsible for antimicrobial activities of 4-(6-methyl-2-naphthyl)-5,6dihydro-6-(substituted phenyl)-oxazine-2amines.

TABLE 1: ANALYTICAL, PHYSICAL CONSTANTS, YIELD AND MASS FRAGMENT OF 4-ARYL-5,6DIHYDRO-6(SUBSTITUTED PHENYL)-4H-1,3-OXAZINE-2-AMINES

\begin{tabular}{|c|c|c|c|c|c|c|}
\hline Entry & $\mathrm{X}$ & M.F. & M.W. & $\begin{array}{c}\text { Yield } \\
(\%)\end{array}$ & m.p. $\left({ }^{\circ} \mathrm{C}\right)$ & Mass $(\mathrm{m} / \mathrm{z})$ \\
\hline 1 & $\mathrm{H}$ & $\mathrm{C}_{21} \mathrm{H}_{20} \mathrm{~N}_{2} \mathrm{O}$ & 317 & 90 & $117-118$ & $317\left[\mathrm{M}^{+}\right], 311,255,175,160,157,91,77,43,42,31,30,16$ \\
\hline 2 & $3-\mathrm{NH}_{2}$ & $\mathrm{C}_{21} \mathrm{H}_{21} \mathrm{~N}_{3} \mathrm{O}$ & 332 & 89 & $107-108$ & $\begin{array}{c}332\left[\mathrm{M}^{+}\right], 316,315,274,255,190,160,157,134,98,92,91,77 \\
58,32,31,16,15\end{array}$ \\
\hline 3 & $4-\mathrm{NH}_{2}$ & $\mathrm{C}_{21} \mathrm{H}_{21} \mathrm{~N}_{3} \mathrm{O}$ & 332 & 89 & $95-96$ & $347\left[\mathrm{M}^{+}\right], 316,274,255,190,160,157,98,92,91,77,31,16,15$ \\
\hline 4 & $3-\mathrm{Br}$ & $\mathrm{C}_{21} \mathrm{H}_{19} \mathrm{BrN}_{2} \mathrm{O}$ & 395 & 87 & $117-118$ & $\begin{array}{c}395\left[\mathrm{M}^{+}\right], 397\left[\mathrm{M}^{2+}\right], 316,301,255,175,157,91,79,77,45,42 \\
31,16\end{array}$ \\
\hline 5 & $3-\mathrm{Cl}$ & $\mathrm{C}_{21} \mathrm{H}_{19} \mathrm{ClN}_{2} \mathrm{O}$ & 351 & 86 & $131-143$ & $\begin{array}{c}351\left[\mathrm{M}^{+}\right], 353\left[\mathrm{M}^{2+}\right], 316,350,335,308,272,255,209,194,157 \\
11,94,91,84,77,58,43,42,35,31,16\end{array}$ \\
\hline 6 & $4-\mathrm{Cl}$ & $\mathrm{C}_{21} \mathrm{H}_{19} \mathrm{ClN}_{2} \mathrm{O}$ & 366 & 86 & $123-124$ & $\begin{array}{c}351\left[\mathrm{M}^{+}\right], 353\left[\mathrm{M}^{2+}\right], 335,308,272,255,111,94,91,84,77,42,35 \\
31,16\end{array}$ \\
\hline 7 & $\begin{array}{c}4- \\
\mathrm{N}\left(\mathrm{CH}_{3}\right)_{2}\end{array}$ & $\mathrm{C}_{23} \mathrm{H}_{25} \mathrm{~N}_{3} \mathrm{O}$ & 360 & 88 & $115-116$ & $\begin{array}{c}360\left[\mathrm{M}^{+}\right], 359,345,344,331,255,218,157,120,102,99,91,77 \\
44,43,31,30,16,15,14\end{array}$ \\
\hline 8 & $4-\mathrm{OH}$ & $\mathrm{C}_{21} \mathrm{H}_{20} \mathrm{~N}_{2} \mathrm{O}_{2}$ & 333 & 87 & $100-101$ & $\begin{array}{c}333\left[\mathrm{M}^{+}\right], 332,331,318,316,255,197,175,157,127,91,77,43 \\
42,31,17,16\end{array}$ \\
\hline 9 & $4-\mathrm{OCH}_{3}$ & $\mathrm{C}_{22} \mathrm{H}_{22} \mathrm{~N}_{2} \mathrm{O}_{2}$ & 347 & 92 & $125-126$ & $\begin{array}{c}347\left[\mathrm{M}^{+}\right], 322,304,255,205,198,164,157,109,107,84,77 \\
58,43,42,31,15,16\end{array}$ \\
\hline 10 & $4-\mathrm{CH}_{3}$ & $\mathrm{C}_{22} \mathrm{H}_{22} \mathrm{~N}_{2} \mathrm{O}$ & 331 & 91 & $107-108$ & $\begin{array}{c}331\left[\mathrm{M}^{+}\right], 330,315,288,255,240,175,157,127,91,84,77,43 \\
42,31,16,15\end{array}$ \\
\hline 11 & $2-\mathrm{NO}_{2}$ & $\mathrm{C}_{21} \mathrm{H}_{19} \mathrm{~N}_{3} \mathrm{O}_{3}$ & 362 & 85 & $117-118$ & $\begin{array}{c}362\left[\mathrm{M}^{+}\right], 361,346,331,319,275,255,198,185,179,164,157 \\
136,127,99,91,77,58,46,43,31,16\end{array}$ \\
\hline 12 & $3-\mathrm{NO}_{2}$ & $\mathrm{C}_{21} \mathrm{H}_{19} \mathrm{~N}_{3} \mathrm{O}_{3}$ & 362 & 86 & $127-128$ & $\begin{array}{c}362\left[\mathrm{M}^{+}\right], 361,346,331,255,179,164,157,136,127,99,91,77, \\
46,43,31,16\end{array}$ \\
\hline 13 & $4-\mathrm{NO}_{2}$ & $\mathrm{C}_{21} \mathrm{H}_{19} \mathrm{~N}_{3} \mathrm{O}_{3}$ & 362 & 85 & $109-110$ & $\begin{array}{c}362\left[\mathrm{M}^{+}\right], 361,346,331,319,275,255,185,179,164,, 136,127, \\
91,77,58,43,31,16\end{array}$ \\
\hline
\end{tabular}


TABLE 2: THE EFFECT OF SOLVENTS IN CONVENTIONAL HEATING AND WITHOUT SOLVENT IN MICROWAVE IRRADIATION ON YIELD OF OXAZINE AMINE (ENTRY 1).

\begin{tabular}{|c|c|c|c|c|}
\hline \multicolumn{4}{|c|}{ Solvents } & \multirow{2}{*}{$\begin{array}{c}\text { Microwave } \\
\text { irradiation }\end{array}$} \\
\hline MeOH & EtOH & DCM & THF & 90 \\
\hline 61 & 60 & 64 & 65 & 9 \\
\hline
\end{tabular}

$\mathrm{MeOH}=$ Methanol; EtOH=Ethanol;

DCM=Dihloro-methane;

$\mathrm{THF}=$ Tetrahydrofuron

Disc size: $6.35 \mathrm{~mm}$; Duration: 24-45 h; Standard: Ampicillin (30-33 $\mathrm{mm})$ and Streptomycin(20-25 mm); Control: Methanol; ---: No activities; \pm : Active(8-12 $\mathrm{mm})$; +: Moderately active $(13-19 \mathrm{~mm})$; ++: Active(20-24 mm).

TABLE 3: THE OXAZINE-2-AMINES 4-6 SHOWED MAXIMUM ZONE OF INHIBITION

AGAINST ESCHERICHIA COLI, WITH GREATER THAN 20 MM COMPARED TO THE OXAZINES 3, 8-10 ARE ACTIVE IN 8-12 MM OF ZONE OF INHIBITION. THE OXAZINES $1,2,7$, 11-13 ARE INACTIVE.

\begin{tabular}{|c|c|c|c|c|c|c|c|}
\hline 氞 & $\mathrm{X}$ & $\begin{array}{l}\dot{\vec{\delta}} \\
\dot{2}\end{array}$ & $\begin{array}{l}\tilde{\Xi} \\
\text { ș } \\
\text { ș }\end{array}$ & 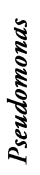 & 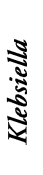 & 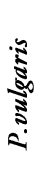 & $\underbrace{5}_{i=1}$ \\
\hline 1 & $\mathrm{H}$ & -- & \pm & $-\cdots$ & \pm & --- & -- \\
\hline 2 & 3- $\mathrm{NH}_{2}$ & -.. & ++ & + & $\ldots$ & \pm & \pm \\
\hline 3 & $4-\mathrm{NH}_{2}$ & \pm & -- & + & ++ & -- & \\
\hline 4 & $3-\mathrm{Br}$ & ++ & + & ++ & ++ & + & ++ \\
\hline 5 & $3-\mathrm{Cl}$ & ++ & \pm & ++ & + & + & + \\
\hline 6 & 4-Cl & ++ & ++ & ++ & + & ++ & ++ \\
\hline 7 & $4-\mathrm{N}\left(\mathrm{CH}_{3}\right)_{2}$ & -- & -- & \pm & \pm & -- & \pm \\
\hline 8 & $4-\mathrm{OH}$ & \pm & -- & \pm & \pm & \pm & \pm \\
\hline 9 & $4-\mathrm{OCH}_{3}$ & \pm & \pm & -.. & -.. & \pm & -. \\
\hline 10 & $4-\mathrm{CH}_{3}$ & \pm & \pm & \pm & \pm & \pm & \pm \\
\hline 11 & $2-\mathrm{NO}_{2}$ & -- & + & + & -. & \pm & -- \\
\hline 12 & $3-\mathrm{NO}_{2}$ & -- & + & --- & \pm & -- & \pm \\
\hline 13 & $4-\mathrm{NO}_{2}$ & -- & + & \pm & --- & + & \\
\hline
\end{tabular}

TABLE 4: ANTIFUNGAL ACTIVITIES OF 4-(6METHYL-2-NAPHTHYL)-5,6-DIHYDRO6(SUBSTITUTED PHENYL)-4H-1,3-OXAZINE-2AMINES

\begin{tabular}{|c|c|c|c|c|}
\hline \multirow{2}{*}{ 氞 } & \multirow[b]{2}{*}{$\mathrm{X}$} & \multirow{2}{*}{ 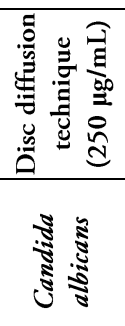 } & \multicolumn{2}{|c|}{ 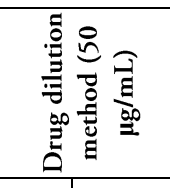 } \\
\hline & & & 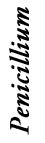 & 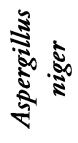 \\
\hline 1 & $\mathrm{H}$ & $-\cdots$ & $-\ldots$ & $-\cdots$ \\
\hline 2 & $3-\mathrm{NH}_{2}$ & \pm & \pm & \pm \\
\hline 3 & $4-\mathrm{NH}_{2}$ & \pm & \pm & \pm \\
\hline 4 & $3-\mathrm{Br}$ & $-\ldots$ & \pm & + \\
\hline 5 & $3-\mathrm{Cl}$ & + & ++ & + \\
\hline 6 & 4-Cl & + & ++ & + \\
\hline 7 & $4-\mathrm{N}\left(\mathrm{CH}_{3}\right)_{2}$ & \pm & \pm & \pm \\
\hline 8 & 4-OH & $-\cdots$ & -- & $-\cdots$ \\
\hline 9 & $4-\mathrm{OCH}_{3}$ & $-\cdots$ & --- & + \\
\hline 10 & $4-\mathrm{CH}_{3}$ & + & + & $-\cdots$ \\
\hline 11 & $2-\mathrm{NO}_{2}$ & ++ & ++ & ++ \\
\hline 12 & $3-\mathrm{NO}_{2}$ & ++ & ++ & ++ \\
\hline 13 & $4-\mathrm{NO}_{2}$ & ++ & ++ & ++ \\
\hline
\end{tabular}

Standard: Griseofulvin and Gentamycin; Duration : 72 h; Control: Methanol; Medium: Potato dextrose agar; ++: No fungal colony; +: One fungal colony; \pm : Two-three fungal colonies; ---: Heavy fungal colony.

\section{CONCLUSIONS}

Some unsaturated 1,3-oxazine-2-amine derivatives including 4-(6-methyl-2naphthyl)-5,6-dihydro-6-(substituted phenyl) -oxazine-2-amines have been synthesised by solvent-free cyclization of 6-methyl-2naphthyl chalcones and urea in presence of hydroxyapatite catalyst under microwave irradiation. The yields of these oxazines were more than $85 \%$. This synthetic methodology offers non-hazardous, solvent-free cyclization, easy-workup procedure, shorter reaction time and better yields. These oxazine derivatives were characterized by their physical constants, spectral data and these data supports the compounds. The antimicrobial activities of these oxazine-2-amines were evaluated using Bauer-Kirby method. The amino, 
dimethylamino, methoxy, methyl, dimethyl, chloro, bromo and nitro substituents responded for the antibacterial and antifungal activities.

\section{REFERENCES}

[1] Cherian, J., Choi, I., Nayyar, A,, Manjunatha, U. H., Mukherjee, T., Lee, Y. S., Boshoff, H. I., Singh, R., Ha, Y. H., Goodwin, M., Lakshminarayana, S. B., Niyomrattanakit, P., Jiricek, J., Ravindran, R., Dick, T., Keller, T. H., Dartois, V., Barry, C. E., Structure-Activity Relationships of Antitubercular Nitroimidazoles. Exploration of the Linker and Lipophilic Tail of ((S)-2-Nitro-6,7-dihydro-5Himidazo[2,1-b][1,3] oxa zin-6-yl)-(4trifluoromethoxybenzyl) amine (6-Amino PA824). J. Med. Chem., 54(16), 5639-5659, 2011.

[2] Thompson, A. M., Sutherland, H. S., Palmer, B. D., Kmentova, I., Blaser, A., Franzblau, S. G., Wan, B., Wang, Y., Ma, Z., Denny, W. A., Synthesis and structure-activity relationships of varied ether linker analogues of the antitubercular drug (6S)-2-nitro-6-\{[4-(trifluoromethoxy) benzyl $]$ oxy\}-6,7-dihydro-5H-imidazo[2,1-

b] $[1,3]$ oxazines, J. Med. Chem., 54, 6563-6585, 2011.

[3] Yakovlev, I. P., Prep'yalov, A. V., Ivin, B. A., Unsaturated 4H-1,3-Oxazines (Review), Chem. Heterocycl. Compd., 30(3), 255-271, 1994.

[4] Manjula, M. K., Rai, K. M. L., Gaonkar, S. L., Raveesha, K. A., Satish, S. Synthesis of new series of 5,6-dihydro-4H-1,2-oxazines via hetero DeilsAlder reaction and evaluation of antimicrobial activity, Eur. J. Med. Chem., 44, 280-288, 2009.

[5] Mathew, B. P., Kumar, A., Sharma, S., Shukla, P. K., Nath, M., An eco-friendly synthesis and antimicrobial activities of dihydro-2H-benzo-and naphtho-1,3-oxazine derivatives, Eur. J. Med. Chem., 45, 1502-1507, 2010.

[6] Elarfi, M. J., Al-Difar, H. A., Synthesis of some heterocyclic compounds derived from chalcones, Sci. Rev. Chem. Commun., 2(2) 103-107, 2012.

[7] Tiwari, V., Meshram, J., Ali, P., Sheikh, J., Tripathi, U., Novel oxazine skeletons as potential antiplasmodial active ingredients: Synthesis, in vitro and in vivo biology of some oxazine entities produced via cyclization of novel chalcone intermediates, J. Enzyme Inhibit. Med. Chem., 26(4), 569-578, 2011.
[8] Das, B. C., Madhukumar, A. V., Anguiano, J., Mani, S., Design, synthesis and biological evaluation of $2 \mathrm{H}$-benzo[b][1,4] oxazine derivatives as hypoxia targeted compounds for cancer therapeutics, Bioorg. Med. Chem. Lett., 19(15), 4204-4206, 2009.

[9] Zhou, D., Harrison, B. L., Shah, U., Andree, T. H., Hornby, G. A., Scerni, R., Studies toward the discovery of the next generation of antidepressants. Part 5: 3,4-Dihydro-2Hbenzo[1,4] oxazine derivatives with dual 5-HT1A receptor and serotonin transporter affinity, Bioorg. Med. Chem. Lett., 16(5), 1338-1341, 2006.

[10] Wang, S., Li, Y., Liu, Y., Lu, A., You, Q., Novel hexacyclic camptothecin derivatives. Part 1: synthesis and cytotoxicity of camptothecins with an A-ring fused 1,3-oxazine ring., Bioorg. Med. Chem. Lett. 18(14), 4095-4097, 2008.

[11] Ando, Y., Ando, K., Yamaguchi, M., Kunitomo, J., Koida, M., Fukuyama, R., A novel oxazine ring closure reaction affording (Z)-((E)-2styrylbenzo[b]furo $\quad[3, \quad 2-d][1,3]$ oxazin-4ylideno)acetaldehydes and their anti-osteoclastic bone resorption activity, Bioorg. Med. Chem. Lett., 16(22), 5849-5654, 2006.

[12] Benameur, L., Bouaziz, Z., Nebois, P., Bartoli, M.H., Boitard, M., Fillion, H., Synthesis of furonaphth[1,3] oxazine and furo[1,3] oxazino quinoline derivatives as precursors for an oquinonemethide structure and potential antitumor agents., Chem. Pharm. Bull. (Tokyo)., 44(3), 605-608,1996.

[13] Roy, K., Mitra, I., Saha, A., Molecular shape analysis of antioxidant and squalene synthase inhibitory activities of aromatic tetrahydro-1,4oxazine derivatives, Chem. Biol. Drug Design., 74(5), 507-516, 2009.

[14] Blaser, A., Palmer, B. D., Sutherland, H. S., Kmentova, I., Franzblau, S. G., Wan, B., Structure-activity relationships for amide-, carbamate-, and urea-linked analogues of the tuberculosis drug (6S)-2-nitro-6-\{[4(trifluoromethoxy) benzyl] oxy\}-6,7-dihydro-5Himidazo[2,1-b][1,3] oxazines, J. Med. Chem., 55(1), 312-326, 2012.

[15] Seal, L., Von Hoff. D., Lawrence, R., Izbicka, E., Jamison, R. M., An in vitro assessment of the antineoplastic potential of 2H-1,3-oxazine2,6(3H)-dione (3-oxauracil), a novel pyrimidine, Invest. New Drugs.,15(4), 289-293, 1997. 
[16] Brudeli, B., Moltzau, L. R., Andressen, K. W., Krobert, K. A., Klaveness, J., Levy, F. O., Synthesis and pharmacological properties of novel hydrophilic 5-HT4 receptor antagonists, Bioorg. Med. Chem., 8(24), 8600-8613, 2010.

[17] Akhter, M., Husain, A., Akhter, N., Khan, M.S.Y., Synthesis, antiinflammatory and antimicrobial activity of some new 1-(3-Phenyl3,4-Dihydro-2H-1,3-Benzoxazin-6-yl)-ethanone derivatives, Indian J. Pharm. Sci., 73, 101-104, 2011.

[18] Gothi, D., Joshi, J. M., Resistant TB: Newer Drugs and Community Approach, Recent Patents and Antiinfectant Drug Discovery., 6(1), 27-37, 2011.

[19] Oh. K. S., Lee, S., Choi, J. K., Lee, B. H., Identification of novel scaffolds for I $\mathrm{B}$ kinase beta inhibitor via a high-throughput screening TR-FRET assay, Comb. Chem. High. Through. Scr., 13(9), 790-797, 2010.

[20] Cho, S. Y., Baek, J. Y., Han. S. S., Kang, S. K., Ha, J. D., Ahn, J. H., PTP-1B inhibitors: cyclopenta[d][1,2]-oxazine derivatives, Bioorg. Med. Chem. Lett., 16(3), 499-502, 2006.

[21] Lee, S. F., Vérolet, Q., Fürstenberg, A., Improved Super-Resolution Microscopy with Oxazine Fluorophores in Heavy Water, Angew. Chem. Inter. Edn., 52(34), 8948-8951, 2013.

[22] Kass L. A., A selective stain for eosinophils using two oxazine dyes applied sequentially, Biotech Histochem., 70(1), 19-23, 1995.

[23] Kass L. A., Identification of neutrophils with an oxazine dye, Biotech Histochem., 70(1), 29-33, 1995.

[24] Jung, C., Müller, B. K., Lamb, D. C., Nolde, F., Müllen, K., Bräuchle, C., A new photostable terrylene diimide dye for applications in single molecule studies and membrane labeling, J. Am. Chem. Soc., 128(15), 5283-5291, 2006.

[25] Verma, A. K., Chioudhary, D., Saunthwal, R. K., Rustagi, V., Patel, M., Tiwari, R. K., Silvercatalyzed domino approach for the synthesis of Benzoxazine/Oxazine fused isoquinolines and naphthyridines from o-alkenyl aldehydes, J. Org. Chem., 78(13), 6657-6669, 2013.

[26] Khalilzadeh, M. A., Yavari, I., Hossaini, Z., Sadeghifar, H., N-Methylimidazole promoted efficient synthesis of 1,3-oxazine-4-thiones under solvent-free conditions, Monatsch Chem.. 140, 467-471, 2009.
[27] Sapkal, S. B., Shelke, K. F., Kategaonkar, A. H., Shingare, M. S.. Dual role of ammonium acetate for solvent-free synthesis of 1,3-disubstituted-2,3dihydro-1H-naphth-[1,2e][1,3]-oxazine, Green Chem. Lett. 2(2), 57-60, 2009.

[28] Thirunarayanan, G., Mayavel, P., Thirumurthy, K., Fly-ash: H2SO4 catalyzed solvent free efficient synthesis of some aryl chalcones under microwave irradiation, Spectrochim. Acta. Part A. 18-22, 91, 2012.

[29] Thirunarayanan, G., Spectral correlation, antimicrobial and insect antifeedant activities of some 1-naphthyl keto-oxiranes, J. Saudi Chem. Soc.. 18, 854-863, 2014,

[30] Arulkumaran, R., Sundararajan, R., Vijayakumar, S., Sakthinathan, S. P., Suresh, R., Kamalakkannan, D., Ranganathan, K., Vanangamudi, G., Thirunarayanan, G., Solvent free synthesis, spectral correlation and antimicrobial activities of some 2E-4nitrochalcones, J. Saudi Chem. Soc. (2012) http://dx. doi.org/10.1016/ j.jscs. 2012.09.006.

[31] Janaki P., Sekar K.G., Thirunarayanan, G., Synthesis, spectral correlation and insect antifeedant activities of some 2-benzimidazole chalcones, J. Saudi Chem. Soc., 20, 58-68, 2016.

[32] McMillan, J. H., Washburne, S. S., Detailed Synthetic Procedure for 4-(4-bromophenyl)1,3(3H) Oxazine-2,6-Dione and related 4 and 5aryl substituted-1,3(3H)Oxazine-2,6-DionesSpectroscopic and analytical data are included, Temple University, (2013).http://www.archive. org.

[33] Turgut, Z., Pelit, E., Koycil, A. K., Synthesis of New 1,3-Disubstituted-2,3-dihydro-1H-naphth$[1,2 \mathrm{e}][1,3]$ oxazines, Molecules., 12, 345-352, 2007.

[34] Subramanian, M., Vanangamudi G., Thirunarayanan, G., Hydroxyapatite catalyzed solvent-free aldol condensation: Synthesis, spectral linearity, antimicrobial and insect antifeedant activities of some 2, 5-dimethyl-3furyl chalcones, Spectro Chim. Acta. Part A., 110, 116-123, 2013

[35] Bauer, A. W., Kirby, W. M. M., Sherris, J. C., Truck, M., Antibiotic Susceptibility Testing by a Standardized Single Disk Method. Am. J. Clin. Pathol., 45, 493-498, 1966.

[36] Thirunarayanan, G., Synthesis, charact- erization and evaluation of biological activities of some 2,3- 
diaryl bicyclo methanones, Ovidius Univ. Annal. Chem., 27(1), 2016, doi: 10.1515/auoc-2016000.

[37] Prabahar S, Balasubramanian V, Suryanarayanan $\mathrm{N}$ and Muthukumarasamy., Optical properties of copper indium diselenide thin films, Chalcogenide letters., 7(1): 49-58, 2010.
[38] Zahr El-Deen $\mathrm{H}$ and Hafez A.I., Physicschemical stability of PVA films doped with $\mathrm{Mn}+2$ ions against weathering conditions, The Arabian Journal for science and engineering., 34(1A): 1326, 2009. 


\title{
التخليق والأنشطة المضادة للميكروبات لبعض
}

\author{
\}

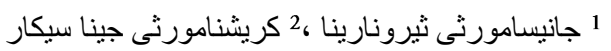 \\ 1 قسم الكيمياء - جامعة أنمالاي - الهند

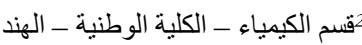

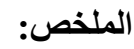

بعض \}4- (6- ميثيل-2-نفثيل) -6،6-ثنائي هيدرو-6- (فينيل مستبدله) - 4 يد 1،3- اوكسازين- 2- أمين $\{$ تم تخليقها باستخدام الدذيبات المحفزة الهيدروكسابتيه والخالية من حلقات 6- ميثيل-2- نفتيل جالكون واليوريا تحت أشعة الميكروويف، وكانت نواتج الاوكسازين أكثر من 85٪. تم تحديد خواص أوكسازين الأمين المخلق و التي تميزت بثبات خواصها الفيزيائية وكذللك بنيانها الطيفية. نم تقييم الأنشطة المضادة للميكروبات لكل التحضير اتر 4- (6- ميثيل-2-نفتيل) -5،6-ثنائي هيدرو-6- (فينيل مستبدله) - 4 يد- 1 ،3 -اوكسازين- 2- أمين\{ باستخدام طريقة الإنتشار الباور-كيربي القرصي مع مجموعة متنو عة من السلالات البكتيرية و الفطرية. 